\title{
ON THE GEOMETRIC PROPERTIES OF NEW TYPE MODULAR SPACE
}

\author{
MEHMET ŞENGÖNÜL
}

\begin{abstract}
In this paper, using a modular, we have defined the modular space $M_{m^{*}}(p)$ and we have shown that the sequence space $M_{m^{*}}(p)$ equipped with the Luxemburg norm is rotund and possesses H-property (or Kadec-Klee property) when $p=\left(p_{k}\right)$ is bounded with $p_{k}>1$ for all $k \in \mathbb{N}$.
\end{abstract}

\section{Introduction and Preliminaries}

Recently, Sanhan and Suantai[11] have generalized normed Cesàro sequence spaces to paranormed sequence spaces by making use of the Köthe sequence spaces. They have defined and studied modular structure and some geometrical properties of these generalized sequence spaces.

As they have done, we have investigated the $M_{m^{*}}(p)$ - modular sequence space and we have shown that $M_{m^{*}}(p)$ - modular sequence space equipped with the Luxemburg norm is rotund and possess $\mathrm{H}$-property (or Kadec-Klee property) when $p=\left(p_{k}\right)$ is bounded with $p_{k}>$ 1 for all $k \in \mathbb{N}$.

Now, let's give some well-known descriptions to understand the subject better.

Let us denote the set of all real numbers and the set of all natural numbers $\mathbb{R}$ and $\mathbb{N}$, respectively. A sequence space is linear subspace of $w$, where $w=\left\{x=\left(x_{k}\right)_{k \in \mathbb{N}}: x: \mathbb{N} \rightarrow\right.$ $\left.\mathbb{R}, k \rightarrow x_{k}=x(k)\right\}$. An $F K$ - space whose topology is normable is called a $B K$ - space [3].

Let $\lambda$ be a subset space of $w$. For a Banach space $\lambda$ we denote by $S(\lambda)$ and $B(\lambda)$ the unit sphere and unit ball of $\lambda$, respectively. A point $x_{0} \in S(\lambda)$ is called:

a) an extreme point if for every $x, y \in S(\lambda)$ the equality $2 x_{0}=x+y$ implies $x=y$;

$b$ ) an H-point if for any sequence $\left(x_{n}\right)$ in $\lambda$ such that $\|x\| \rightarrow 1$ as $n \rightarrow \infty$, the weak convergence of $\left(x_{n}\right)$ to $x_{0}$ implies that $\left\|x_{n}-x\right\| \rightarrow 0$ as $n \rightarrow \infty$.

A Banach space $\lambda$ is said to be rotund, if every point of $S(\lambda)$ is an extreme point. A Banach space $\lambda$ is said to possess H-property(or Kadec-Klee property) provided every point of $S(\lambda)$ is an H-point. 
Let $\lambda$ be an arbitrary vector space over $\mathbb{C}$. In this case:

(a) A functional $m: \lambda \rightarrow[0, \infty]$ is called modular if the following conditions hold:

(M1) $m(x)=0 \Leftrightarrow x=0$,

(M2) $m(r x)=m(x)$ for $r \in \mathbb{R}$ (or $\mathbb{C}$ ) with $|r|=1$, for all $x \in \lambda$,

(M3) $m(r x+s y) \leq m(x)+m(y)$ if $r, s \geq 0, r+s=1$, for all $x, y \in \lambda$.

(b) If $M 3$ is replaced by;

(M4) $m(r x+s y)=r^{\mu} m(x)+s^{\mu} m(y)$ if $r, \quad s \geq 0, \quad r^{\mu}+s^{\mu}=1$, with an $\mu \in[0,1]$ then the modular $m$ is called an $\mu$-convex modular, and if $\mu=1, m$ is called a convex modular.

(c) modular $m$ defines the corresponding modular space, i.e, the space $\lambda_{m}$ given by $\lambda_{m}=$ $\{x \in w: m(t x) \rightarrow 0$ as $t \rightarrow \infty\}$.

Recall that for given any $\epsilon>0$ a sequence $\left(x_{n}\right)$ is said to be an $\epsilon$-separated sequence if $\operatorname{sep}\left(x_{n}\right)=\inf \left\{\left\|x_{n}-x_{k}\right\|: n \neq k\right\}>\epsilon$. We say a Banach space $\lambda$ has $\beta$-property if for every $\epsilon>0$ such that, for each element $x_{0} \in B(\lambda)$ and each sequence $\left(x_{n}\right) \in B(\lambda)$ with $\operatorname{sep}\left(x_{n}\right) \geq \epsilon$, there exists an index $k$ such that $\left\|\frac{x_{0}+x_{k}}{2}\right\| \leq 1-\delta$.

The Nakano sequence space $\ell(p)$ is defined by

$$
\ell(p)=\left\{x=\left(x_{k}\right) \in w: m(t x)<\infty \text { for some } t>0\right\},
$$

where $m(x)=\sum_{k}\left|x_{k}\right|^{p_{k}}$ and $p=\left(p_{k}\right)$ is a sequence of positive real numbers with $p_{k} \geq 1$ for all $k \in \mathbb{N}$. The space $\ell(p)$ is a Banach space with the norm

$$
\|x\|_{\ell(p)}=\inf \left\{t>0: m\left(\frac{x}{t}\right) \leq 1\right\} .
$$

If $p=\left(p_{k}\right)$ is bounded, we have

$$
\ell(p)=\left\{x=\left(x_{k}\right) \in w: \sum_{k}\left|x_{k}\right|^{p_{k}}<\infty\right\} .
$$

Also, some geometric properties of $\ell(p)$ were studied in [2] and [4].

For $1 \leq p<\infty$, the Cesàro sequence space is defined by

$$
\operatorname{ces}_{p}=\left\{x=\left(x_{k}\right) \in w:\left(\sum_{n}\left(\frac{1}{n} \sum_{k=1}^{n}\left|x_{k}\right|\right)^{p}\right)^{\frac{1}{p}}<\infty\right\}
$$

equipped with the norm

$$
\|x\|=\left(\sum_{n}\left(\frac{1}{n} \sum_{k=1}^{n}\left|x_{k}\right|\right)^{p}\right)^{\frac{1}{p}}
$$


This space was introduced by Shue [12]. Some geometric properties of sequence spaces $c e s_{p}$ and $\ell(p)$ have been thoroughly discussed by many mathematicians in the literature. It is known that ces $_{p}$ is locally uniform rotund and possess H-property [8]. Cui and Hudzik [4] proved that $c e s_{p}$ has the Banach-Saks of type $p$ if $p>1$, and it was shown in [5] that ces $_{p}$ has $\beta$-property. Also, we know that Nakano sequence spaces are special cases of Musielak- Orlicz sequence spaces. Some results in this paper may give some ideas of how geometric properties of general Musielak- Orlicz sequence spaces can be obtained. In this paper, we extend the study to another sequence space which we have described below. The space $\operatorname{ces}(p)[10]$ is defined by

$$
\operatorname{ces}(p)=\{x \in w: \rho(t x)<\infty \text { for some } t>0\}
$$

where $\rho(x)=\sum_{n}\left(\frac{1}{n+1} \sum_{k=0}^{n}\left|x_{k}\right|\right)^{p_{n}}$. The space $\operatorname{ces}(p)$ is a Banach space with the norm

$$
\|x\|=\inf \left\{t>0: \rho\left(\frac{x}{t}\right) \leq 1\right\}
$$

and if $p=\left(p_{n}\right)$ is bounded then we have

$$
\operatorname{ces}(p)=\left\{x \in w: \sum_{n}\left(\frac{1}{n+1} \sum_{k=0}^{n}\left|x_{k}\right|\right)^{p_{n}}<\infty\right\} .
$$

Several geometric properties of $\operatorname{ces}(p)$ have already been studied in [10].

Subsequently the relation between modular sequence spaces and the sequence spaces defined by Orlicz function have been discussed by various authors: (Lindenstrauss, J. and Tzafriri, L.,[7], Y. Altin; M. Et and B.C. Tripathy [1], B. C. Tripathy and S. Mahanta [14], M. Et; Y. Altin; B. Choudhary and B.C. Tripathy [6], B.C. Tripathy, Y. Altin and M. Et [15], B.C. Tripathy and B. Sarma [16], B.C. Tripathy and S. Borgogain [17], B.C. Tripathy and B. Sarma [18], B.C. Tripathy and H. Dutta [19], B.C. Tripathy and B. Hazarika: [20], B.C. Tripathy and B. Sarma [21], B.C. Tripathy and P. Chandra [22])

Quite recently, Şimşek and Karakaya [13] have defined the sequence space $\ell_{\rho}(u, v, p)$ as

$$
\ell_{\rho}(u, v, p)=\{x \in w: \rho(\lambda x) \leq \infty, \text { for some } \lambda>0\}
$$

where

$$
\rho(x)=\sum_{k}\left(u_{k} \sum_{j=0}^{k} v_{j}\left|x_{j}\right|\right)^{p_{k}}
$$

and $u, v \in U=\left\{u \in w: u_{k}>0\right.$ for all $\left.k \in \mathbb{N}\right\}$. In[13], they investigated some geometric and topological properties of the space $\ell_{\rho}(u, v, p)$ such as Kadec-Klee property and modular structure of $\ell_{\rho}(u, v, p)$, uniform opial property, etc. 
In the same way we can introduce the new type modular sequence space $M_{m^{*}}(p)$ as follows:

$$
M_{m^{*}}(p)=\left\{x=\left(x_{k}\right) \in w: m^{*}(\lambda x)<\infty, \text { for some } \lambda>0\right\}
$$

where

$$
m^{*}(x)=\sum_{k}\left(\frac{u_{k}}{v_{k}} \sum_{j=0}^{k} w_{j}\left|x_{j}\right|\right)^{p_{k}}
$$

and $u, v, w \in U$. The so-called Luxemburg norm on the sequence space $M_{m^{*}}(p)$ is defined as follows:

$$
\|x\|_{M_{m^{*}}(p)}=\inf \left\{t>0: m^{*}\left(\frac{x}{t}\right) \leq 1\right\} .
$$

If $p=\left(p_{k}\right)$ is bounded, then we have

$$
M_{m^{*}}(p)=\left\{x \in w: \sum_{k}\left(\frac{u_{k}}{v_{k}} \sum_{j=0}^{k} w_{j}\left|x_{j}\right|\right)^{p_{k}}<\infty\right\} .
$$

The purpose of this note is to define and to investigate the $M_{m^{*}}(p)$ - modular sequence space and show that $M_{m^{*}}(p)$ - modular sequence space equipped with the Luxemburg norm is rotund and possesses H-property (or Kadec-Klee property) when $p=\left(p_{k}\right)$ is bounded with $p_{k}>1$ for all $k \in \mathbb{N}$. Furthermore, throughout this paper we assume that $p=\left(p_{i}\right)$ is bounded with $p_{i}>1$ for all $i \in \mathbb{N}$ and $H=\sup _{i} p_{i}$ and $K=\max \left\{1,2^{H-1}\right\}$.

Clearly, in the special case $\left(v_{k}\right)=(e)=(1,1,1, \ldots, 1, \ldots)$ we have $M_{m^{*}}(p)=\ell_{\rho}(u, v, p)$ normed by

$$
\|x\|=\inf \left\{\lambda>0: \sum_{k}\left(u_{k} \sum_{j=0}^{k} v_{j}\left|\frac{x_{j}}{\lambda}\right|\right)^{p_{k}} \leq 1\right\}
$$

and if we take $\left(v_{k}\right)=(k+1),\left(u_{k}\right)=\left(w_{k}\right)=(e)$ for all $k \in \mathbb{N}$ then the space $M_{m^{*}}(p)$ reduces to the modular space $\operatorname{Ces}(p)[9]$ normed by

$$
\|x\|_{\operatorname{Ces}(p)}=\inf \left\{\lambda>0: \sum_{k}\left(\frac{1}{k+1} \sum_{j=0}^{k}\left|\frac{x_{j}}{\lambda}\right|\right)^{p_{k}} \leq 1\right\} .
$$

Furthermore, if we take $\left(u_{k}\right)=(e),\left(w_{k}\right)=\left(p_{k}\right),\left(v_{k}\right)=\left(P_{k}\right)$ and $P_{k}=\sum_{i=0}^{k} p_{i}$ for all $i, j \in \mathbb{N}$ then the space $M_{m^{*}}(p)$ reduces to the modular space $N_{\rho}(p)$ [23] normed by

$$
\|x\|=\inf \left\{\lambda>0: \sum_{k}\left(\frac{1}{P_{k}} \sum_{j=0}^{k} p_{j}\left|\frac{x_{j}}{\lambda}\right|\right)^{p_{k}} \leq 1\right\},
$$


i.e., the spaces $\ell_{\rho}(u, v, p), N_{\rho}(p)$ and $\operatorname{Ces}(p)$ are very special cases of the modular space $M_{m^{*}}(p)$

\section{Modular structure of $M_{m^{*}}(p)$}

Let us begin by proving the following theorem which about isomorphism:

Theorem 2.1. The sequence spaces $M_{m^{*}}(p)$ is linearly isomorphic to the space $\ell(p)$.

Proof. We should show the existence of a linear bijection between the spaces $M_{m^{*}}(p)$ and $\ell(p)$. Consider the transformation $T$ define from $M_{m^{*}}(p)$ to $\ell(p)$ by $T: M_{m^{*}}(p) \rightarrow \ell(p), x \rightarrow$ $T x=y, \quad y=\left(y_{k}\right), \quad y_{k}=\frac{u_{k}}{v_{k}} \sum_{j=0}^{k} w_{j} x_{j},(i \in \mathbb{N})$. The linearity of $T$ is clear. Further, it is trivial that $x=0$ whenever $T x=0$ and hence $T$ is injective. Let $y \in \ell(p)$ and define the sequence $x=\left(x_{k}\right)$ by

$$
x_{k}=\frac{1}{w_{k}}\left[\frac{v_{k}}{u_{k}} y_{k}-\frac{v_{k-1}}{u_{k-1}} y_{k-1}\right] \quad(k \in \mathbb{N}) .
$$

Then

$$
\begin{aligned}
\|x\|_{M_{m^{*}}(p)} & =\inf \left\{t>0: m^{*}\left(\frac{x}{t}\right) \leq 1\right\}=\inf \left\{t>0: \sum_{k}\left(\frac{u_{k}}{v_{k}} \sum_{j=0}^{k} \frac{w_{j}}{t}\left|x_{j}\right|\right)^{p_{k}} \leq 1\right\} \\
& =\inf \left\{t>0: \sum_{k}\left(\frac{u_{k}}{v_{k}} \sum_{j=0}^{k} \frac{w_{j}}{t}\left|\frac{1}{w_{j}}\left[\frac{v_{j}}{u_{j}} y_{j}-\frac{v_{j-1}}{u_{j-1}} y_{j-1}\right]\right|\right)^{p_{k}} \leq 1\right\} \\
& =\inf \left\{t>0: \sum_{k}\left(\left|\frac{y_{k}}{t}\right|\right)^{p_{k}} \leq 1\right\}=\|y\|_{\ell(p)}
\end{aligned}
$$

Thus, we have that $x \in M_{m^{*}}(p)$ and consequently $T$ is surjective. Hence, $T$ is linear bijection which therefore says us that the spaces $M_{m^{*}}(p)$ and $\ell(p)$ are linearly isomorphic. This completes proof.

Theorem 2.2. The functional $m^{*}$ on the sequence space $M_{m^{*}}(p)$ is a convex modular.

Proof. Let $x, y \in M_{m^{*}}(p)$. It is clear that $m^{*}(x)=0 \Leftrightarrow x=0$ and $m^{*}(r x)=m^{*}(x)$ for all scalar $r$ with $|r|=1$, so we omit it. Again, let's suppose that $x, y \in M_{m^{*}}(p)$ and $r \geq 0, s \geq 0$ with $r+s=1$. By the convexity of the function $u \rightarrow|u|^{p_{n}} ; n \in \mathbb{N}$, we have:

$$
\begin{aligned}
m^{*}(r x+s y)= & \sum_{k}\left(\frac{u_{k}}{v_{k}} \sum_{j=0}^{k} w_{j}\left|r x_{j}+s y_{j}\right|\right)^{p_{k}} \\
& \leq \sum_{k}\left(\sigma_{k} \sum_{j=0}^{k} u_{k} v_{j}\left|r x_{j}\right|+\frac{u_{k}}{v_{k}} \sum_{j=0}^{k} w_{j}\left|s y_{j}\right|\right)^{p_{k}}
\end{aligned}
$$




$$
\begin{aligned}
& =\sum_{k}|r|^{p_{k}}\left(\frac{u_{k}}{v_{k}} \sum_{j=0}^{k} w_{j}\left|x_{j}\right|\right)^{p_{k}}+\sum_{k}|s|^{p_{n}}\left(\frac{u_{k}}{v_{k}} \sum_{j=0}^{k} w_{j}\left|s y_{j}\right|\right)^{p_{k}} \\
& \leq r \sum_{k}\left(\frac{u_{k}}{v_{k}} \sum_{j=0}^{k} w_{j}\left|x_{j}\right|\right)^{p_{k}}+s \sum_{k}\left(\frac{u_{k}}{v_{k}} \sum_{j=0}^{k} w_{j}\left|y_{j}\right|\right)^{p_{k}} \\
& =r m^{*}(x)+s m^{*}(y) .
\end{aligned}
$$

Theorem 2.3. For $x \in M_{m^{*}}(p)$ the modular $m^{*}$ on $M_{m^{*}}(p)$ satisfies the following properties:

1. If $0<t<1$ then $t^{K} m^{*}\left(\frac{x_{j}}{t}\right) \leq m^{*}(x)$ and $m^{*}(t x) \leq t m^{*}(x)$,

2. If $t>1$, then $m^{*}(x) \leq t^{K} m^{*}\left(\frac{x_{j}}{t}\right)$,

3. If $t \geq 1$, then $m^{*}(x) \leq t m^{*}(x) \leq m^{*}(t x)$.

\section{Proof.}

1. If $0<t<1$ then we have

$$
\begin{aligned}
m^{*}(x) & =\sum_{k}\left(\frac{u_{k}}{v_{k}} \sum_{j=0}^{k} w_{j}\left|x_{j}\right|\right)^{p_{k}}=\sum_{k}\left(t \frac{u_{k}}{v_{k}} \sum_{j=0}^{k} w_{j}\left|\frac{x_{j}}{t}\right|\right)^{p_{k}} \\
& =\sum_{k} t^{p_{k}}\left(\frac{u_{k}}{v_{k}} \sum_{j=0}^{k} w_{j}\left|\frac{x_{j}}{t}\right|\right)^{p_{k}} \geq \sum_{k} t^{K}\left(\frac{u_{k}}{v_{k}} \sum_{j=0}^{k} w_{j}\left|\frac{x_{j}}{t}\right|\right)^{p_{k}} \\
& =t^{K} \sum_{k}\left(\frac{u_{k}}{v_{k}} \sum_{j=0}^{k} w_{j}\left|\frac{x_{j}}{t}\right|\right)^{p_{k}}=t^{K} m^{*}\left(\frac{x_{j}}{t}\right),
\end{aligned}
$$

and if we consider convexity of $m^{*}$ then we see that $m^{*}(t x) \leq t m^{*}(x)$. Hence 1 is satisfied. 2. Let $t>1$ then,

$$
\begin{aligned}
m^{*}(x) & =\sum_{k}\left(\frac{u_{k}}{v_{k}} \sum_{j=0}^{k} w_{j}\left|x_{j}\right|\right)^{p_{k}}=\sum_{k} t^{p_{k}}\left(\frac{u_{k}}{v_{k}} \sum_{j=0}^{k} w_{j}\left|\frac{x_{j}}{t}\right|\right)^{p_{k}} \\
& \leq t^{K} \sum_{k}\left(\frac{u_{k}}{v_{k}} \sum_{j=0}^{k} w_{j}\left|\frac{x_{j}}{t}\right|\right)^{p_{k}}=t^{K} m^{*}\left(\frac{x_{j}}{t}\right)
\end{aligned}
$$

so 2 is obtained.

3. It is obvious that 3 is satisfied by the convexity of $m^{*}$.

Now, let's talk about the relationships between the Luxemburg norm and the modular $m^{*}$ on the space modular sequence space $M_{m^{*}}(p)$.

Theorem 2.4. For any $x \in M_{m^{*}}(p)$, if $p=\left(p_{n}\right)$ is bounded, we have

4. If $\|x\|_{M_{m^{*}}(p)}<1$ then $m^{*}(x) \leq\|x\|_{M_{m^{*}}(p)}$

5. If $\|x\|_{M_{m^{*}}(p)}>1$ then $m^{*}(x) \geq\|x\|_{M_{m^{*}}(p)}$ 


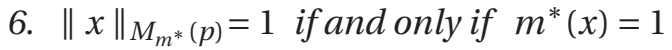

7. $\|x\|_{M_{m^{*}}(p)<1}$ if and only if $m^{*}(x)<1$

8. $\|x\|_{M_{m^{*}}(p)}>1$ if and only if $m^{*}(x)>1$

9. If $0<r<1$ and $\|x\|_{M_{m^{*}}(p)}>r$ then $m^{*}(x)>r^{K}$

10. If $r \geq 1$ and $\|x\|_{M_{m^{*}}(p)}<r$ then $m^{*}(x)<r^{K}$

\section{Proof.}

4. Let $\epsilon>0$ be such that $0<\epsilon<1-\|x\|_{M_{m^{*}}(p)} \Rightarrow\|x\|_{M_{m^{*}}(p)}+\epsilon<1$. From definition of \|\|$_{M_{m^{*}}(p)}$ there exists $t>0$ such that $\|x\|_{M_{m^{*}}(p)}+\epsilon>t$ and accordingly $\frac{\|x\|_{M_{m^{*}}(p)}+\epsilon}{t}>1$ and $m^{*}\left(t^{-1} x\right) \leq 1$. From Theorem 2.3 (1. and 3.) we have

$$
m^{*}(x) \leq m^{*}\left(\left(\|x\|_{M_{m^{*}}(p)}+\epsilon\right) t^{-1} x\right) \leq\left(\|x\|_{M_{m^{*}}(p)}+\epsilon\right) m^{*}\left(t^{-1} x\right) \leq\|x\|_{M_{m^{*}}(p)}+\epsilon
$$

which implies that $m^{*}(x) \leq\|x\|_{M_{m^{*}}(p)}$. So 4 is satisfied.

5. If $0<\epsilon<\left(\|x\|_{M_{m^{*}}(p)}-1\right)\|x\|_{M_{m^{*}}(p)}^{-1}$ then $1<(1-\epsilon)\|x\|_{M_{m^{*}}(p)}<\|x\|_{M_{m^{*}}(p)}$ and by definition of $\|\cdot\|_{M_{m^{*}}(p)}$ with by part 1 of Theorem 2.3 we have

$$
1<m^{*}\left(x\left[(1-\epsilon)\|x\|_{M_{m^{*}}(p)}\right]^{-1}\right) \leq\left[(1-\epsilon)\|x\|_{M_{m^{*}}(p)}\right]^{-1} m^{*}(x) .
$$

So $(1-\epsilon)\|x\|_{M_{m^{*}}(p)}<m^{*}(x)$ for all $\epsilon \in\left(0,\left(\|x\|_{M_{m^{*}}(p)}-1\right)\|x\|_{M_{m^{*}}(p)}^{-1}\right)$. This implies that $\|$ $x \|_{M_{m^{*}}(p)} \leq m^{*}(x)$, hence 5 is obtained.

6. We have that $m^{*}(x)=1$ implies that $\|x\|_{M_{m^{*}}(p)}=1$. Let's suppose that $\|x\|_{M_{m^{*}}(p)}=1$. By the definition of $\|x\|_{M_{m^{*}}(p)}$ we have that for any $\epsilon>0$ there exists $\mu>0$ such that $1+\epsilon>\mu>\|$ $x \|_{M_{m^{*}}(p)}$ and $m^{*}\left(x \mu^{-1}\right) \leq 1$. By part 2 of Theorem 2.3, we have

$$
m^{*}(x) \leq \mu^{K} m^{*}\left(x \mu^{-1}\right) \leq \mu^{K}<(1+\epsilon)^{K} .
$$

By this way $\left(m^{*}(x)\right)^{K^{-1}}<1+\epsilon$ for all $\epsilon>0$, which implies $m^{*}(x) \leq 1$. If $m^{*}(x)<1$, then we can choose $r \in(0,1)$ such that $m^{*}(x)<r^{K}<1$. If we consider 1 of Theorem 2.3, we have $m^{*}\left(r^{-1} x\right) \leq\left(r^{K}\right)^{-1} m^{*}(x)<1$. Hence, $\|x\|_{M_{m^{*}}(p)} \leq r<1$ which is a contradiction. Therefore $m^{*}(x)=1$.

7. The proof is clear from 4 . and 6.

8. The proof is obvious from 6 . and 7.

9. Suppose $0<r<1$ and $\|x\|_{M_{m^{*}}(p)}>r$. Then $\left\|x r^{-1}\right\|_{M_{m^{*}}(p)}>1$. By 5 we have $m^{*}\left(x r^{-1}\right)>1$. Hence, from 1 of Theorem 2.3, we obtain that $m^{*}(x) \geq r^{K} m^{*}\left(r^{-1} x\right)>r^{K}$.

10. Suppose that $r \geq 1$ and $\|x\|_{M_{m^{*}}(p)}<r$. Then $\left\|x r^{-1}\right\|_{M_{m^{*}}(p)}<1$. From 7 we have $\left\|x r^{-1}\right\|_{M_{m^{*}}(p)}<$ 1. If $r=1$, it is obvious that $m^{*}(x)<1=r^{K}$. If $r>1$ then by part2 of Theorem 2.3; we obtain that $m^{*}(x) \leq r^{K} m^{*}\left(r^{-1} x\right)<r^{K}$. 
Theorem 2.5. Let $\left(x_{n}\right)$ be a sequence in $M_{m^{*}}(p)$, where $p=\left(p_{k}\right)$ is bounded. Then;

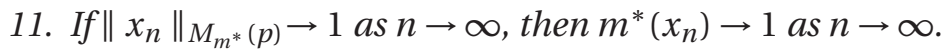

12. If $m^{*}\left(x_{n}\right) \rightarrow 0$ as $n \rightarrow \infty$, then $\left\|x_{n}\right\|_{M_{m^{*}}(p)} \rightarrow 0$ as $n \rightarrow \infty$.

\section{Proof.}

11. Let's suppose that $\|x\|_{M_{m^{*}}(p)} \rightarrow 1$ as $n \rightarrow \infty$ and let $\epsilon \in(0,1)$. Then there exists $N \in \mathbb{N}$ such that $1-\epsilon<\left\|x_{n}\right\|_{M_{m^{*}}(p)}<1+\epsilon$ for all $n \in \mathbb{N}$. By Theorem 2.4 we have $(1-\epsilon)^{K}<m^{*}\left(x_{n}\right)<(1+\epsilon)^{K}$ for all $n \geq N$ which implies $m^{*}\left(x_{n}\right) \rightarrow 1$ as $n \rightarrow \infty$.

12. Now, let us suppose that $\left\|x_{n}\right\|_{M_{m^{*}}(p)} \rightarrow 0$ as $n \rightarrow \infty$. Then there must be an $\epsilon \in(0,1)$ and a subsequence $\left(x_{n_{k}}\right)$ of $\left(x_{n}\right)$ such that $\left\|x_{n_{k}}\right\|_{M_{m^{*}}(p)}>\epsilon$ for all $k \in \mathbb{N}$. By part 9. of Theorem 2.4 we have $m^{*}\left(x_{n_{k}}\right)>\epsilon^{K}$ for all $k \in \mathbb{N}$. This implies $m^{*}\left(x_{n_{k}}\right) \rightarrow 0$ as $n \rightarrow \infty$.

Theorem 2.6. The $M_{m^{*}}(p)$-Nakano sequence space is a Banach space with the norm defined by (1.5).

\section{Proof.}

Clearly, (1.5) is a norm on the space $M_{m^{*}}(p)$ and the remaining portion of the work can be proved by using the techniques similar to those used in Theorem 2.4. of Şimşek and Karakaya [13].

\section{Kadec Klee property of the space $M_{m^{*}}(p)$}

Let us now give the following interesting theorem which we will use about the Kadec-Klee property of the space $M_{m^{*}}(p)$.

Theorem 3.7. Let $x \in M_{m^{*}}(p)$ and $\left(x^{k}\right) \subseteq M_{m^{*}}(p)$. If $\lim _{k} m^{*}\left(x^{n}\right)=m^{*}(x)$ and $\lim _{n} x_{i}^{n}=x_{i}$ for all $i \in \mathbb{N}$ then $\lim _{n} x^{n}=x$ in $M_{m^{*}}(p)$, that is $\left\|x^{n}-x\right\|_{M_{m^{*}}(p)} \rightarrow 0$ as $n \rightarrow \infty$.

Proof. Let $\epsilon>0$ be given. Then there is $n_{0} \in \mathbb{N}$ such that

$$
\sum_{k=n_{0}+1}^{\infty}\left(\frac{u_{k}}{v_{k}} \sum_{j=0}^{k} w_{j}\left|x_{j}\right|\right)^{p_{k}}<\epsilon(6 K)^{-1}
$$

since $m^{*}(x)=\sum_{k}\left(\frac{u_{k}}{v_{k}} \sum_{j=0}^{k} w_{j}\left|x_{j}\right|\right)^{p_{k}}<\infty$. Since

$$
\lim _{n}\left[m^{*}\left(x^{n}\right)-\sum_{k=0}^{n_{0}}\left(\frac{u_{k}}{v_{k}} \sum_{j=0}^{k} w_{j}\left|x_{j}^{n}\right|\right)^{p_{k}}\right]=m^{*}(x)-\sum_{k=0}^{n_{0}}\left(\frac{u_{k}}{v_{k}} \sum_{j=0}^{k} w_{j}\left|x_{j}\right|\right)^{p_{k}}
$$

and $\lim _{n} x_{j}^{n}=x_{j}, \forall j \in \mathbb{N}$, there is $n_{0} \in \mathbb{N}$ such that

$$
\left|\sum_{k=k_{0}+1}^{\infty}\left(\frac{u_{k}}{v_{k}} \sum_{j=0}^{k} w_{j}\left|x_{j}^{n}\right|\right)^{p_{k}}-\sum_{k=k_{0}+1}^{\infty}\left(\frac{u_{k}}{v_{k}} \sum_{j=0}^{k} w_{j}\left|x_{j}\right|\right)^{p_{k}}\right|<(3 K)^{-1}
$$


and

$$
\sum_{k=0}^{k_{0}}\left(\frac{u_{k}}{v_{k}} \sum_{j=0}^{k} w_{j}\left|x_{j}^{n}-x_{j}\right|\right)^{p_{k}}<3^{-1} \epsilon
$$

That for $n \geq n_{0}$ and from (3.1), (3.2) and (3.3)

$$
\begin{aligned}
& m^{*}\left(x^{n}-x\right)=\sum_{k=0}^{\infty}\left(\frac{u_{k}}{v_{k}} \sum_{j=0}^{k} w_{j}\left|x_{j}^{n}-x_{j}\right|\right)^{p_{k}}=\sum_{k=0}^{k_{0}}\left(\frac{u_{k}}{v_{k}} \sum_{j=0}^{k} w_{j}\left|x_{j}^{n}-x_{j}\right|\right)^{p_{k}} \\
& +\sum_{k=k_{0}+1}^{\infty}\left(\frac{u_{k}}{v_{k}} \sum_{j=0}^{k} w_{j}\left|x_{j}^{n}-x_{j}\right|\right)^{p_{k}} \\
& <3^{-1} \epsilon+K\left[\sum_{k=k_{0}+1}^{\infty}\left(\frac{u_{k}}{v_{k}} \sum_{j=0}^{k} w_{j}\left|x_{j}^{n}\right|\right)^{p_{k}}\right. \\
& \left.+\sum_{k=k_{0}+1}^{\infty}\left(\frac{u_{k}}{v_{k}} \sum_{j=0}^{k} w_{j}\left|x_{j}\right|\right)^{p_{k}}\right] \\
& =3^{-1} \epsilon+K\left[m^{*}\left(x^{n}\right)-\sum_{k=0}^{k_{0}}\left(\frac{u_{k}}{v_{k}} \sum_{j=0}^{k} w_{j}\left|x_{j}^{n}\right|\right)^{p_{k}}\right. \\
& \left.+\sum_{k=k_{0}+1}^{\infty}\left(\frac{u_{k}}{v_{k}} \sum_{j=0}^{k} w_{j}\left|x_{j}^{n}\right|\right)^{p_{k}}\right] \\
& <3^{-1} \epsilon+K\left[m^{*}(x)-\sum_{k=0}^{k_{0}}\left(\frac{u_{k}}{v_{k}} \sum_{j=0}^{k} w_{j}\left|x_{j}\right|\right)^{p_{k}}\right. \\
& \left.+(3 K)^{-1} \epsilon+\sum_{k=k_{0}+1}^{\infty}\left(\frac{u_{k}}{v_{k}} \sum_{j=0}^{k} w_{j}\left|x_{j}\right|\right)^{p_{k}}\right] \\
& =3^{-1} \epsilon+K\left[\sum_{k=k_{0}+1}^{\infty}\left(\frac{u_{k}}{v_{k}} \sum_{j=0}^{k} w_{j}\left|x_{j}\right|\right)^{p_{k}}\right. \\
& \left.+(3 K)^{-1} \epsilon+\sum_{k=k_{0}+1}^{\infty}\left(\frac{u_{k}}{v_{k}} \sum_{j=0}^{k} w_{j}\left|x_{j}\right|\right)^{p_{k}}\right] \\
& =3^{-1} \epsilon+K\left[\epsilon K^{-1}+2 \sum_{k=k_{0}+1}^{\infty}\left(\frac{u_{k}}{v_{k}} \sum_{j=0}^{k} w_{j}\left|x_{j}\right|\right)^{p_{k}}\right] \\
& =3^{-1} \epsilon+K\left[\epsilon(3 K)^{-1}+2 \epsilon(6 K)^{-1}\right] \\
& <3^{-1} \epsilon+3^{-1} \epsilon+3^{-1} \epsilon=\epsilon
\end{aligned}
$$

This shows that $m^{*}\left(x^{n}-x\right) \rightarrow 0$ as $n \rightarrow \infty$. And consequently by part 12 of Theorem 2.5 we have that $\left\|x^{n}-x\right\|_{M_{m^{*}}(p)} \rightarrow 0$ as $k \rightarrow \infty$ and this completes the proof.

Theorem 3.8. The space $M_{m^{*}}(p)$ has the H-property (or Kadec-Klee property). 


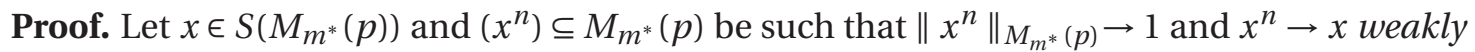
as $n \rightarrow \infty$.

From Theorem 2.2, we have $m^{*}(x)=1$ so it follows from Theorem 2.3 that $m^{*}\left(x^{n}\right) \rightarrow m(x)$ as $n \rightarrow \infty$. Since the mapping $p_{i}: M_{m^{*}}(p) \longrightarrow \mathbb{R}$, defined by $p_{i}(y)=y_{i}$ is a continuous linear functional on $M_{m^{*}}(p)$ it implies that $x_{i}^{n} \rightarrow x_{i}$ as $n \rightarrow \infty$ for all $i \in \mathbb{N}$. Thus, by Theorem 3.7, we get $x^{n} \rightarrow x$ as $n \rightarrow \infty$.

Theorem 3.9. If $p=\left(p_{k}\right)$ is bounded then the $M_{m^{*}}(p)$ is rotund .

Proof. Let $x \in S\left(M_{m^{*}}(p)\right)$ and $y, z \in B\left(M_{m^{*}}(p)\right)$ with $x=2^{-1}(y+z)$. Since convexity of $m^{*}$ and from Theorem 2.4 we have

$$
1=m^{*}(x) \leq 2^{-1}\left[m^{*}(y)+m^{*}(z)\right] \leq 2^{-1}(1+1),
$$

so that $m^{*}(x)=2^{-1}\left[m^{*}(y)+m^{*}(z)\right]=1$. This suggests that

$$
\left(\frac{u_{k}}{v_{k}} \sum_{j=0}^{k} w_{j}\left|2^{-1}\left(y_{j}+z_{j}\right)\right|\right)^{p_{k}}=2^{-1}\left(\frac{u_{k}}{v_{k}} \sum_{j=0}^{k} w_{j}\left|\left(y_{j}\right)\right|\right)^{p_{k}}+2^{-1}\left(\frac{u_{k}}{v_{k}} \sum_{j=0}^{k} w_{j}\left|z_{j}\right|\right)^{p_{k}}
$$

for all $n \in \mathbb{N}$. We shall show that $y_{k}=z_{k}$ for all $k \in \mathbb{N}$. For $k=0$ from (3.4) we have

$$
\left|x_{0}\right|^{p_{0}}=2^{-1}\left[\left|y_{0}\right|^{p_{0}}+\left|z_{0}\right|^{p_{0}}\right] .
$$

Since the mapping $u \rightarrow|u|^{p_{0}}$ is strictly convex, from (3.5) it is implied that $y_{0}=z_{0}$. Now assume that $y_{i}=z_{i}$ for all $i=1,2, \ldots, k-1$. Then $y_{i}=z_{i}=x_{i}$ for all $i=1,2, \ldots, k-1$. From (3.4) we have

$$
\begin{gathered}
\left(\frac{u_{k}}{v_{k}} \sum_{j=0}^{k} w_{j}\left|2^{-1}\left(y_{j}+z_{j}\right)\right|\right)^{p_{k}}=\left(2^{-1}\left[\frac{u_{k}}{v_{k}} \sum_{j=0}^{k} w_{j}\left|x_{j}+y_{j}\right|\right]\right)^{p_{k}} \\
=2^{-1}\left(\sigma_{k} \sum_{j=0}^{k} u_{k} v_{j}\left|y_{j}\right|\right)^{p_{k}}+2^{-1}\left(\frac{u_{k}}{v_{k}} \sum_{j=0}^{k} w_{j}\left|z_{j}\right|\right)^{p_{k}}
\end{gathered}
$$

By the convexity of the mapping $u \rightarrow|u|^{p_{k}}, \sigma_{k} \sum_{j=0}^{k} u_{k} v_{j}\left|y_{j}\right|=\frac{u_{k}}{v_{k}} \sum_{j=0}^{k} w_{j}\left|z_{j}\right|$ is implied. Since $y_{k}=z_{k}$ for all $i=1,2, \ldots, k-1$ we get that

$$
\left|y_{k}\right|=\left|z_{k}\right|
$$

If $y_{k}=0$, then we have $y_{k}=z_{k}=0$. Suppose that $y_{k} \neq 0$. Then $z_{k} \neq 0$. If $y_{k} z_{k}<0$ it follows from (3.8) that $y_{k}+z_{k}=0$. This implies that (3.6) and (3.8)

$$
\left(\frac{u_{k}}{v_{k}} \sum_{j=0}^{k} w_{j}\left|x_{j}\right|\right)^{p_{k}}=\left(\frac{u_{k}}{v_{k}} \sum_{j=0}^{k} w_{j}\left|x_{j}\right|+\frac{u_{k}}{v_{k}} \sum_{j=0}^{k} w_{j}\left|y_{j}\right|\right)^{p_{k}}
$$


which is a contradiction. Thus, we have $y_{k} z_{k}>0$. This shows that, by (3.5) $y_{k}=z_{k}$. Thus, from the principle of mathematical induction, we have $y_{k}=z_{k}$ for all $n \in \mathbb{N}$, so $y=z$.

\section{Acknowledgements}

The author would like to express his pleasure to referee(s) for his/her careful reading and making some useful corrections on the first draft of this paper which improved the presentation and readability.

\section{References}

[1] Y. Altin, M. Et and B. C. Tripathy, The sequence space $|\bar{N} p|(M, r, q, s)$ on seminormed spaces, Applied Mathematics and Computation 154(2004), 423-430.

[2] S. T. Chen, Geometry of Orlicz spaces, Dissertationes Math., 1996, pp. 356.

[3] B. Cohuldhary and S. Nanda, Functional Analysis with Applications, John Wiley \& Sons Inc. New Delhi. 1989.

[4] Y. A. Cui, H. Hudzik and R. Pluciennik, Banach Saks property in some Banach sequence spaces, Ann. Math. Polinici 65(1997), 193-202.

[5] Y. A. Cui and C. Meng, Banach-Saks property ( $\beta$ ) in Cesàro sequence spaces, SEA. Bull. Math. Tamkang J. Math. 24(2000), 201-210.

[6] M. Et, Y. Altin, B. Choudhary and B. C. Tripathy, On some classes of sequences defined by sequences of Orlicz functions, Mathematical Inequalities and Applications, 9(2)(2006), 335-342.

[7] Lindenstrauss, J. and Tzafriri, L., On Orlicz sequence classes, Israel J. Math. 10 (1971), 379-390.

[8] Y. Q. Lui, B. E. Wu and Y. P. Lee, Method of sequence spaces, Guangdong of science and Technology Press, (in Chine) 1996.

[9] N. Petrot and S. Suantai, Uniform opial properties in generalized Cesàro sequence spaces, Nonlinear Analysis: Theory, Methods and Applications 63(2005), 1116-1125.

[10] W. Sanhan, On geometric properties some Banach sequence spaces, Thesis for the degree of Master of Science in Mathematics, Chiang Mai University, 2000.

[11] W. Sanhan and S. Suantai, Some geometric properties of Cesàro sequence space, Kyungpook Mathematical Journal,43(2003), 191-197.

[12] J. S. Shue, Cesàro sequence spaces, Tamkang J. Math. 1(1970), 143-150.

[13] N. Şimşek and V. Karakaya On some geometrical properties of generalized modular spaces of Cesàro type defined by weighted means, Journal of Inequalities and Applications, (2009).

[14] B. C. Tripathy and S. Mahanta, On a class of generalized lacunary difference sequence spaces defined by Orlicz function, Acta Mathematica Applicata Sinica, 20(2004), 231-238.

[15] B. C. Tripathy, Y. Altin and M. Et, Generalized difference sequences spaces on seminormed spaces defined by Orlicz functions, Mathematica Slovaca, 58(2008), 315-324.

[16] B. C. Tripathy and B. Sarma, Sequence spaces of fuzzy real numbers defined by Orlicz functions, Mathematica Slovaca, 58(2008), 621-628.

[17] B. C. Tripathy and S. Borgogain, The sequence space $m\left(M, f, \Delta_{m}^{n}, p\right)^{F}$, Mathematical Modelling and Analysis 13(2008), 577-586.

[18] B. C. Tripathy and B. Sarma, Vector valued double sequence spaces defined by Orlicz function, Mathematica Slovaca 59(2009), 767-776.

[19] B. C. Tripathy and H. Dutta, On some new paranormed difference sequence spaces defined by Orlicz functions, Kyungpook Mathematical Journal 50(2010), 59-69.

[20] B. C. Tripathy and B. Hazarika, I-convergent sequences spaces defined by Orlicz function, Acta Mathematica Applicatae Sinica 27(2011) 149-154. 
[21] B. C. Tripathy and B. Sarma, Double sequence spaces of fuzzy numbers defined by Orlicz function, Acta Mathematica Scientia 31B(2011), 134-140.

[22] B. C. Tripathy and P. Chandra, On some generalized difference paranormed sequence spaces associated with multiplier sequences defined by modulus function, Anal. Theory Appl. 27(2011), 21-27.

[23] C. S. Wang, On Nörlund sequence spaces, Tamkang Journal of Mathematics, 9(1978), 269-274.

Faculty of Arts and Sciences, Department of Mathematics, Nevşehir Üniversitesi, 2000 Evler Mah. Züeyde Hanım Cad. 50300 NEVŞEHİR.

E-mail: msengonul@yahoo.com 